\title{
Portfolio Selection in the Credibilistic Framework Using Renyi Entropy and Renyi Cross Entropy
}

\author{
Gholanhossein Yari $^{1}$, Alireza Sajedi ${ }^{1}$, and Mohamadtaghi Rahimi ${ }^{2}$ \\ ${ }^{1}$ Department of Statistics, Islamic Azad University, Science and Research Branch, Tehran, Iran \\ ${ }^{2}$ Department of Mathematics, Iran University of Science and Technology, Tehran, Iran
}

\section{]jfis}

\begin{abstract}
In this study, the Renyi entropy-mean-variance maximization and Renyi cross entropy-meanvariance minimization models for portfolio selection with fuzzy return were investigated under the credibility theory framework. We also attempted to examine the relationship between credibilistic Renyi entropy-mean-variance and Renyi cross entropy-mean-variance models. The effect of the $v$ parameter in Renyi entropy and Renyi cross entropy on optimal portfolio selection was considered using the presented models.
\end{abstract}

Keywords: Portfolio selection, Credibility theory, Renyi entropy, Renyi cross entropy

\section{Introduction}

Markowitz [1] was the first scientist to introduce the modern portfolio selection theory. The first measure he used to study investment returns was variance, and he relied on logic that suggested the bigger the variance was, the higher the risk would be. Later, many researchers revised or developed the model with new methods or elements to improve the results. Yu et al. [2] pointed out that different risk measurement models lead to significantly different impacts on portfolio structure.

A major advancement in the literature was the argument that the concept of entropy could be used to calculate the risk measure of a portfolio, as long as the risk is taken as an element of uncertainty in portfolio selection. Entropy was first used by Philippatos and Wilson [3] in

Received: Jan. 27, 2018

Revised : Mar. 8, 2018

Accepted: Mar. 21, 2018

Correspondence to: Alireza Sajedi

(alireza.sajedi@srbiau.ac.ir)

(CThe Korean Institute of Intelligent Systems

cCThis is an Open Access article distributed under the terms of the Creative Commons Attribution Non-Commercial License (http://creativecommons.org/licenses/ by-nc/3.0// which permits unrestricted noncommercial use, distribution, and reproduction in any medium, provided the original work is properly cited. portfolio selection, and the mean-entropy model they proposed has become the foundation for research in this field. Moreover, Zhou [4] found that entropy has been more and more widely used in the financial field. Ke and Zhang [5] used the Shannon entropy to modify the mean-variance. Usta and Kantar [6] also used the mean-variance-skewness-entropy model for portfolio diversification, and they found that the models with an entropy factor performed the best in out-of-sample testing. Yu et al. [7] compared the mean-variance efficiency of the models that incorporated different entropy measures by applying the multiple criteria method, and Yari et al. [8] employed entropy optimization measures to determine the weights of each criterion of growth and reduction to better predict the future worth of each share.

Recently, portfolio selection problems have been studied under fuzzy environment conditions. By using the fuzzy set theory, many scholars, including Tanaka and Guo [9], Huang [10, 11], and Abiyev and Menekay [12], took the security returns as fuzzy variables. 
The concept of fuzzy entropy was proposed by $\mathrm{Li}$ and Liu [13] for measuring the uncertainty of fuzzy variables. Huang [10, 11] extended Kapur's entropy maximization model [14] to a fuzzy environment and formulated the mean-entropy model.

This study attempts to improve portfolio selection models by focusing on credibilistic entropy $\alpha$ order maximization and cross entropy $\alpha$ order minimization models. We established credibilistic Renyi entropy maximization and Renyi cross entropy minimization models for portfolio optimization with fuzzy returns in the framework of credibility theory. The main contents include the definition of entropy of fuzzy variables, and the formulation and equivalents of entropy maximization and cross entropy minimization models.

\section{Preliminaries}

The credibility theory, introduced by Liu [15], is a branch of mathematics that studies the behavior of fuzzy phenomena. Let $\theta$ be a nonempty set and $\mathcal{P}$ is a power set of $\Theta$. Any members of the set $\mathcal{P}$ is considered as an event. Li and Liu [16] defined the credibility measure as a mean of possibility measure with the self-duality property. The axioms defined by them for the credibility measure are as follows:

Axiom 1 (Normality). $\operatorname{Cr}\{\Theta\}=1$.

Axiom 2 (Monotonicity). $C r\{A\} \leq C r\{B\}$ whenever $A \subset$ $B$.

Axiom 3 (Self-duality). $C r$ is self-dual, i.e., $C r\{A\}+C r\{A c\}=$ 1 for any event A.

Axiom 4 (Countable subadditivity). $\operatorname{Cr} \bigcup_{i} A_{i}=\sup _{i} \operatorname{Cr}\left\{A_{i}\right\}$ for any events $\left\{A_{i}\right\}$ with $\sup _{i} \operatorname{Cr}\left\{A_{i}\right\}<0.5$.

If the set function, $\mathrm{Cr}$, follows the aforementioned four axioms, then the triple $(\Theta, \mathcal{P}, C r)$ will be called the credibility pace. Let $\xi$ be a fuzzy variable with the $\mu$ membership function. For any $A$ set of real numbers, we obtain

$$
C r(\xi \in A)=\frac{1}{2}\left(\sup _{x \in A} \mu(x)+1-\sup _{x \in A^{c}} \mu(x)\right) .
$$

In credibility theory, Liu and Liu [17] defined the expected value and variance with a finite expected value of fuzzy variable $\xi$ as follows:

$$
E[\xi]=\int_{0}^{\infty} C r(\xi \geq r) d r-\int_{-\infty}^{0} C r(\xi \leq r) d r
$$

$$
\operatorname{Var}(\xi)=E\left[(\xi-E[\xi])^{2} .\right.
$$

If $\xi=\tau(a-\alpha, a, a+\beta)$ is a triangular fuzzy variable with following membership function

$$
\mu(x)= \begin{cases}\frac{(x-a+\alpha)}{\alpha}, & \text { if } a-\alpha \leq x \leq a, \\ \frac{(a+\beta-x)}{\beta}, & \text { if } a \leq x \leq a+\beta, \\ 0, & \text { otherwise. }\end{cases}
$$

The Li and Liu [16] study proved that $E[\xi]=a+\frac{\beta-\alpha}{4}$ and $\operatorname{var}[\xi]=\left\{\begin{array}{l}\frac{33 \alpha^{3}+21 \alpha^{2} \beta+11 \alpha \beta^{2}-\beta^{3}}{384 \alpha} \text { if } \alpha \geq \beta, \\ \frac{33 \beta^{3}+21 \alpha \beta^{2}+11 \alpha^{2} \beta-\alpha^{3}}{384 \beta} \text { if } \alpha \leq \beta .\end{array}\right.$

Definition 1. Let $\xi$ be a continuous fuzzy variable that takes values in $[a, b]$; $\mathrm{Li}$ and $\mathrm{Liu}[13]$ presented the following definition for fuzzy entropy

$$
H[\xi]=\int_{a}^{b} S(C r\{\xi=x\}) d x,
$$

where $S(t)=-t \ln t-(1-t) \ln (1-t)$.

Considering the continuity of the fuzzy variable, we have

$$
0 \leq H[\xi] \leq(b-a) \ln 2 .
$$

Definition 2 (Li [18]). Let $\xi$ and $\eta$ be a continuous fuzzy variable that takes values in $[a, b]$. Then, the cross entropy of $\xi$ and $\eta$ is defined as

$$
D=[\xi ; \eta]=\int_{a}^{b} T(C r\{\xi=x ; C r\{\eta=x\}) d x,
$$

where $T(s ; t)=s \ln \left(\frac{s}{t}\right)+(1-s) \ln \left(\frac{(1-s)}{(1-t)}\right)$.

It is easy to verify that (a) $T(s ; t)$ is strictly convex with respect to $(s, t)$ and attains its minimum value of zero on the line $s=t$; and (b) for any $0 \leq s \leq 1$ and $0 \leq t \leq 1$, we have $T(s ; t)=T(1-s ; 1-t)$.

\section{The Order $v$ Fuzzy Entropy and Cross En- tropy}

Shannon [19] defined the entropy of a probability distribution $\left(p_{1}, p_{2}, \ldots, p_{n}\right)$ as $-\sum_{i=1}^{n} p_{i} \ln p_{i}$. In case of a duality system, the Shannon entropy is shortened to $-p \ln p-(1-p) \ln (1-p)$, where $0 \leq p \leq 1$. This shows the uncertainty of dual-state systems. Appling this concept in a fuzzy environment, the ambiguity measure with $\mu$ membership can be defined as $g(\mu)=$ 
$-\mu \ln \mu-(1-\mu) \ln (1-\mu)$. De Luca and Termini [20] introduced principles for generating fuzzy entropy, where the fuzzy entropy formula for a finite universal set $X=\left\{x_{1}, x_{2}, \ldots, x_{n}\right\}$ is drawn as follows:

$$
\begin{aligned}
E(A)= & -k \sum_{i=1}^{n}\left[\mu_{A}\left(x_{i}\right) \ln \mu_{A}\left(x_{i}\right)\right. \\
& \left.+\left(1-\mu_{A}\left(x_{i}\right)\right) \ln \left(1-\mu_{A}\left(x_{i}\right)\right)\right], \quad k>0 .
\end{aligned}
$$

Renyi [21] defined the order $v$ entropy of a probability distribution $\left(p_{1}, p_{2}, \ldots, p_{n}\right)$ as $\left.\frac{1}{1-v} \ln \sum_{i=1}^{n} p_{i}^{v}\right), v>0$. A similar analysis was used by Bhandari and $\mathrm{Pal}[22]$ to define the order $v$ entropy for a fuzzy set as

$$
\begin{aligned}
& E_{v}(A)=\frac{1}{1-v} \sum_{i=1}^{n} \ln \left[\mu_{A}\left(x_{i}\right)^{v}+\left(1-\mu_{A}\left(x_{i}\right)\right)^{v}\right], \\
& v>0, v \neq 0
\end{aligned}
$$

where $\lim _{v \rightarrow 1} E_{v}(A)=E(A)$.

Taking into account the above points and Definition 1, we introduce a definition of order $v$ fuzzy entropy as follows:

$$
H[\xi]=\int_{a}^{b} S(C r\{\xi=x\}) d x,
$$

where $S_{v}(t)=\frac{1}{1-v} \ln \left[t^{v}+(1-t)^{v}\right], v>0, v \neq 0$.

Considering $\frac{\partial^{2} S_{v}}{\partial t^{2}}<0$, we can conclude that $S_{v}$ is a strictly concave function and that its minimum value is at point zero and its maximum value at point $=\frac{1}{2}$.

Also, based on the definition of Li [18], the fuzzy cross entropy of $\xi$ from $\eta$ is defined as

$$
D=[\xi ; \eta]=\int_{a}^{b} T(C r\{\xi=x ; C r\{\eta=x\}) d x,
$$

where $T(s ; t)=\frac{1}{v-1} \log \left[t^{v} s^{1-v}+(1-t)^{v}(1-s)^{1-v}\right]$.

Theorem 1. Let $\xi=\tau(a-\alpha, a, a+\beta)$ be a triangular fuzzy variable. Then, we obtain the following:

$$
H[\xi]=\frac{\alpha+\beta}{2^{v}(1-v)} \int_{0}^{1} \ln \left[x^{v}+(1-x)^{v}\right] d x .
$$

Proof. Considering Eqs. (9) and (10), we obtain the following:

$$
\begin{aligned}
& H[\xi]=\frac{\alpha+\beta}{2^{v}(1-v)} \int_{0}^{1} \ln \left[\mu(x)^{v}+(1-\mu(x))^{v}\right] d x, \\
& H[\xi]=\frac{1}{2^{v}(1-v)} \int_{a-\alpha}^{a} \ln \left[\left(\frac{x-a+\alpha}{\alpha}\right)^{v}\right.
\end{aligned}
$$

$$
\begin{aligned}
& \left.+\left(1-\frac{x-a+\alpha}{\alpha}\right)^{v}\right] d x \\
& +\frac{1}{2^{v}(1-v)} \int_{a}^{a+\beta} \ln \left[\left(\frac{a+\beta-x}{\beta}\right)^{v}\right. \\
& \left.+\left(1-\frac{a+\beta-x}{\beta}\right)^{v}\right] d x .
\end{aligned}
$$

By the changes of variable technique, we obtain the following:

$$
\begin{aligned}
H[\xi]= & \frac{\alpha}{2^{v}(1-v)} \int_{0}^{1} \ln \left[x^{v}+(1-x)^{v}\right] d x \\
& +\frac{\beta}{2^{v}(1-v)} \int_{0}^{1} \ln \left[x^{v}+(1-x)^{v}\right] d x .
\end{aligned}
$$

Therefore, the theorem is proved, and solving this integral numerically for different values of $v,(v>0)$ is possible.

Theorem 2. Let $\xi=\tau(a-\alpha, a, a+\beta)$ be a triangular fuzzy variable, and $\eta$ is an equipossible fuzzy variable on $[a-\alpha, a+\beta]$. Then, we obtain the following:

$$
D[\xi ; \eta]=\frac{\alpha+\beta}{2(1-v)} \int_{0}^{1} \ln \left[x^{v}+(2-x)^{v}\right] d x .
$$

Proof. Let $\mu$ and $\kappa$ be the membership functions of $\xi$ and $\eta$, respectively. Because $C r\{\xi=x\}=\frac{\mu(x)}{2}$ and $C r\{\eta=x\}=$ $\frac{\kappa(x)}{2}$, the cross entropy of $\xi$ from $\eta$ can be rewritten as

$$
\begin{aligned}
D[\xi ; \eta]= & \frac{1}{\alpha-1} \ln \int_{a}^{b}\left[\left(\frac{\mu(x)}{2}\right)^{v}\left(\frac{\kappa(x)}{2}\right)^{1-v}\right. \\
& \left.+\left(1-\frac{\mu(x)}{2}\right)^{v}\left(1-\frac{\kappa(x)}{2}\right)^{1-v}\right] d x .
\end{aligned}
$$

Considering the $\kappa(x) \equiv 1$ for any $x \in[a, b]$, we obtain the following:

$$
D=[\xi ; \eta]=\frac{1}{2(v-1)} \ln \int_{a}^{b}\left[(\mu(x))^{v}+(2-\mu(x))^{1-v}\right] d x .
$$

By Definitions 1 and 2, we obtain

$$
\begin{aligned}
D[\xi ; \eta]= & \frac{1}{2(1-v)} \ln \int_{a-\alpha}^{a}\left(\frac{x-a+\alpha}{\alpha}\right)^{v} \\
& +\left(2-\frac{x-a+\alpha}{\alpha}\right)^{1-v} d x \\
& +\frac{1}{2(1-v)} \ln \int_{a}^{a+\beta}\left(\frac{a+\beta-x}{\beta}\right)^{v} \\
& +\left(2-\frac{a+\beta-x}{\beta}\right)^{1-v} d x
\end{aligned}
$$


By the changes of variable technique, we obtain the following:

$$
D[\xi ; \eta]=\frac{\alpha+\beta}{2(1-v)} \int_{0}^{1} \ln \left[x^{v}+(2-x)^{1-v}\right] d x .
$$

Therefore, the theorem is proved, and solving this integral numerically for different values of $v,(v>0)$ is possible.

\section{Credibilistic Entropy and Cross-Entropy Or- der $v$ Maximization}

In this section, we introduce the order $v$ maximization entropy model for optimizing a portfolio with fuzzy returns. Let $\xi_{i}$ be the $i$ th return of the security, and $x_{i}$ is the proportion of capital allocated for the ith security, where $i=1,2, \ldots, n$. Let $\xi_{1}, \xi_{2}, \ldots, \xi_{n}$ be the fuzzy variables in the credibility space $(\Theta, \mathcal{P}, C r)$. We seek to build a credibilistic order $v$ maximum entropy model to select the appropriate risk measure and return in order to optimize the portfolio. By changing the $v$ value, we can examine the effects of this value in selecting an optimum portfolio. Let $\xi_{1} x_{1}+\xi_{2} x_{2}+\cdots+\xi_{n} x_{n}$ be a symmetric fuzzy return, and we use variance as a risk measure and propose the following credibilistic entropy order $v$ maximization model,

$$
\left\{\begin{array}{cc}
\max & H\left[\xi_{1} x_{1}+\xi_{2} x_{2}+\cdots+\xi_{n} x_{n}\right] \\
\text { s.t. } & E\left[\xi_{1} x_{1}+\xi_{2} x_{2}+\cdots+\xi_{n} x_{n}\right] \geq r_{0}, \\
& V\left[\xi_{1} x_{1}+\xi_{2} x_{2}+\cdots+\xi_{n} x_{n}\right] \leq d_{0}, \\
& x_{1}+x_{2}+\cdots+x_{n}=1, \\
& x_{i} \geq 0, \quad i=1,2, . ., n,
\end{array}\right.
$$

where $r_{0}$ is the predetermined expected return and $d_{0}$ is the predetermined risk for the portfolio.

Theorem 3. Suppose that $\xi_{i}=\tau\left(a_{i}-\alpha_{i}, a_{i}, a_{i}+\beta_{i}\right)$ are independent triangular fuzzy variables for $i=1,2, \ldots, n$. Then, the above model is equivalent to the crisp model,

$$
\begin{cases}\max & \frac{\left(\alpha_{i}+\beta_{i}\right) x_{i}}{2^{v}(1-v)} \int_{0}^{1} \ln \left[x_{i}{ }^{v}+\left(1-x_{i}\right)^{v}\right] d x \\ \text { s.t. } \quad & \int_{i=1}^{n}\left(4 a_{i}+\beta_{i}-\alpha_{i}\right) x_{i} \geq 4 r_{0}, \\ \sum_{i=1}^{n} \sum_{j=1}^{n}\left(17 \alpha_{i} \alpha_{j}+17 \beta_{i} \beta_{j}+11 \alpha_{i} \beta_{j}+11 \alpha_{j} \beta_{i}\right) x_{i} x_{j} \\ \quad \times\left|\sum_{k=1}^{n}\left(\alpha_{k}-\beta_{k}\right) x_{k}\right| \\ +\sum_{i=1}^{n} \sum_{j=1}^{n} \sum_{k=1}^{n} 16\left(\alpha_{i} \alpha_{j}+\beta_{i} \beta_{j}\right)\left(\alpha_{k}+\beta_{k}\right) x_{i} x_{j} x_{k}\end{cases}
$$

81 | Gholanhossein Yari, Alireza Sajedi, and Mohamadtaghi Rahimi

$$
\left\{\leq 192 d_{0}\left(\sum_{i=1}^{n} x_{i}\left(\alpha_{i}+\beta_{i}\right)+\left|\sum_{i=1}^{n} x_{i}\left(\alpha_{i}-\beta_{i}\right)\right|\right) .\right.
$$

Proof. Because $\xi_{1}, \xi_{2}, \ldots, \xi_{n}$ are independent triangular fuzzy variables, the theorem extension principle of Zade, Liu [15], tell us that

$$
\sum_{i=1}^{n} \xi_{i} x_{i}=\tau\left(\sum_{i=1}^{n}\left(a_{i}-\alpha_{i}\right) x_{i}, \sum_{i=1}^{n} a_{i} x_{i}, \sum_{i=1}^{n}\left(a_{i}+\beta_{i}\right) x_{i}\right)
$$

is also a triangular fuzzy variable. Thus, the expected value and variance of $\xi_{1} x_{1}+\xi_{2} x_{2}+\cdots+\xi_{n} x_{n}$ are $\sum_{i=1}^{n} a_{i} x_{i}+$ $\frac{1}{4} \sum_{i=1}^{n} \beta_{i} x_{i}-\frac{1}{4} \sum_{i=1}^{n} \alpha_{i} x_{i}$ and

$$
\begin{aligned}
& \frac{11\left(\sum_{i=1}^{n} x_{i}\left(\alpha_{i}+\beta_{i}\right)^{2}\left|\sum_{i=1}^{n} x_{i}\left(\alpha_{i}-\beta_{i}\right)\right|\right)}{192\left(\sum_{i=1}^{n} x_{i}\left(\alpha_{i}+\beta_{i}\right)+\left|\sum_{i=1}^{n} x_{i}\left(\alpha_{i}-\beta_{i}\right)\right|\right)} \\
& +\frac{\left(\begin{array}{c}
2\left(8 \sum_{i=1}^{n} x_{i}\left(\alpha_{i}+\beta_{i}\right)+3\left|\sum_{i=1}^{n} x_{i}\left(\alpha_{i}-\beta_{i}\right)\right|\right) \\
\times\left(\left(\sum_{i=1}^{n} x_{i} \alpha_{i}\right)^{2}+\left(\sum_{i=1}^{n} x_{i} \beta_{i}\right)^{2}\right)
\end{array}\right)}{192\left(\sum_{i=1}^{n} x_{i}\left(\alpha_{i}+\beta_{i}\right)+\left|\sum_{i=1}^{n} x_{i}\left(\alpha_{i}-\beta_{i}\right)\right|\right)} .
\end{aligned}
$$

After rearranging these two expressions, the desired results are easy to obtain. Therefore, the theorem is proved.

In addition, we obtain the credibilistic cross entropy order $v$ minimization model, as follows:

$$
\left\{\begin{array}{cr}
\min & D\left[\xi_{1} x_{1}+\xi_{2} x_{2}+\cdots+\xi_{n} x_{n} ; \eta\right] \\
s . t . & E\left[\xi_{1} x_{1}+\xi_{2} x_{2}+\cdots+\xi_{n} x_{n}\right] \geq r_{0} \\
& V\left[\xi_{1} x_{1}+\xi_{2} x_{2}+\cdots+\xi_{n} x_{n}\right] \leq d_{0} \\
& x_{1}+x_{2}+\cdots+x_{n}=1 \\
& x_{i} \geq 0, \quad i=1,2, . ., n
\end{array}\right.
$$

where $r_{0}$ is the predetermined expected return and $d_{0}$ is the predetermined risk for the portfolio.

The crisp model for the above model, as in a proof of Theorem 3. By comparing the models (15) and (18), it can be concluded that there is no difference in optimization portfolio.

\section{Conclusions}

In the present study, we compared the applicability of two models, Renyi entropy-mean-variance and Renyi cross entropymean-variance, for portfolio selection under the credibility theory framework. We showed that there is no difference between the two models as a choice investors. It was also presented that changing the values of the $v$ parameter in the Renyi entropy- 
mean-variance maximization and Renyi cross entropy-meanvariance minimization would not affect the decision of an investor. We may also conclude that using different values of $v$ in proposed models for portfolio optimization would not affect the decision of an investor to allocate his capital to purchase various securities. Eventually, the application of different entropy measures instead of Renyi entropy in the presented models will lead to a single result under the credibility theory for portfolio optimization.

\section{Conflict of Interest}

No potential conflict of interest relevant to this article was reported.

\section{References}

[1] H. Markowitz, "Portfolio selection," The Journal of Finance, vol. 7, no. 1, pp. 77-91, 1952. https://doi.org/10. 1111/j.1540-6261.1952.tb01525.x

[2] M. Yu, R. X. Zhou, and M. Wu, "Study on the model selection in portfolio management," The Journal of Quantitative \& Technical Economics, vol. 30. pp. 98-110, 2013.

[3] G. C. Philippatos and C. J. Wilson, "Entropy market risk and the selection of efficient portfolios," Applied Economics, vol. 4, no. 3, pp. 209-220, 1972. https://doi.org/ $10.1080 / 00036847200000017$

[4] R. Zhou, R. Cai, and G. Tong, "Application of entropy in finance: a review," Entropy, vol. 15, no. 11, pp. 4909-4931, 2013. https://doi.org/10.3390/e15114909

[5] J. Ke and C. Zhang, "Study on the optimization of portfolio based on entropy theory and mean-variance model," in Proceedings of the IEEE/SOLI 2008. IEEE International Conference on Service Operations and Logistics, and Informatics, Beijing, China, 2008, pp. 2668-2672. https://doi.org/10.1109/SOLI.2008.4682988

[6] I. Usta and Y. M. Kantar, "Mean-variance-skewnessentropy measures: a multi-objective approach for portfolio selection," Entropy, vol. 13, no. 1, pp. 117-133, 2011. https://doi.org/10.3390/e13010117

[7] J. R. Yu, W. Y. Lee, W. J. P. Chiou, "Diversified portfolios with different entropy measures," Applied Mathematics and Computation, vol. 241, pp. 47-63, 2014. https://doi. org/10.1016/j.amc.2014.04.006
[8] G. Yari, M. Rahimi, and P. Kumar, "Multi-period multicriteria (MPMC) valuation of American options based on entropy optimization principles," Iranian Journal of Science and Technology, Transactions A: Science, vol. 41, no. 1, pp. 81-86, 2017. https://doi.org/10.1007/s40995017-0206-0

[9] H. Tanaka and P. Guo, "Portfolio selection based on upper and lower exponential possibility distributions," European Journal of Operational Research, vol. 114, no. 1, pp. 115126, 1999. https://doi.org/10.1016/S0377-2217(98)000332

[10] X. Huang, "Mean-entropy models for fuzzy portfolio selection," IEEE Transactions on Fuzzy Systems, vol. 16, no. 4, pp. 1096-1101, 2008. https://doi.org/10.1109/TFUZZ. 2008.924200

[11] X. Huang, "Mean-semivariance models for fuzzy portfolio selection," Journal of Computational and Applied Mathematics, vol. 217, no. 1, pp. 1-8, 2008. https://doi. org/10.1016/j.cam.2007.06.009

[12] R. H. Abiyev and M. Menekay, "Fuzzy portfolio selection using genetic algorithm," Soft Computing, vol. 11, no. 12, pp. 1157-1163, 2007. https://doi.org/10.1007/s00500-007$0157-\mathrm{z}$

[13] P. Li and B. Liu, "Entropy of credibility distributions for fuzzy variables," IEEE Transactions on Fuzzy Systems, vol. 16, no. 1, pp. 123-129, 2008. https://doi.org/10.1109/ TFUZZ.2007.894975

[14] J. Kapur and H. Kesavan, Entropy Optimization Principles with Applications. New York, NY: Academic Press, 1992.

[15] B. Liu, Uncertainty Theory (2nd ed.). Heidelberg: Springer, 2007. https://doi.org/10.1007/978-3-540-731658_5

[16] X. Li and B. Liu, "Maximum entropy principle for fuzzy variables," International Journal of Uncertainty, Fuzziness and Knowledge-Based Systems, vol. 15(Suppl. 2), pp. 4353, 2007. https://doi.org/10.1142/S0218488507004595

[17] B. Liu and Y. K. Liu, "Expected value of fuzzy variable and fuzzy expected value models," IEEE Transactions on Fuzzy Systems, vol. 10, no. 4, pp. 445-450, 2002. https: //doi.org/10.1109/TFUZZ.2002.800692 
[18] Xiang Li, "Fuzzy cross-entropy," Journal of Uncertainty Analysis and Applications, vol. 3, article no. 2, 2015. https: //doi.org/10.1186/s40467-015-0029-5

[19] C. E. Shannon, "A mathematical theory of communication," Bell System Technical Journal, vol. 21, no. 3, pp. 379-423, 1948. https://doi.org/10.1002/j.1538-7305.1948. tb01338.x

[20] A. De Luca and S. Termini, "A definition of a nonprobabilistic entropy in the setting of fuzzy sets theory," Information Control, vol. 20, pp. 301-312, 1972. https://doi.org/10.1016/S0019-9958(72)90199-4

[21] A. Renyi, "On measures of entropy and information," in Proceedings of the 4th Berkeley Symposium on Mathematical Statistics and Probability, Berkeley, CA, 1960, pp. 547-561.

[22] D. Bhandari and N. R. Pal, "Some new information measure of fuzzy sets," Information Sciences, vol. 67, no. 3, pp. 209-228, 1993. https://doi.org/10.1016/00200255(93)90073-U

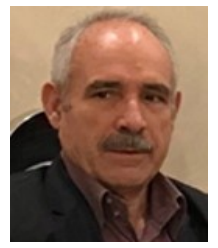

Gholanhossein Yari is an assistant professor in the Department of Applied Mathematics, School of Mathematics at Iran University of Science and Technology. His research interest include information theory, statistics and stochastic processes, several of his recent work focusing on entropy and its application in statistics. Dr. Yari received his Ph.D. in Applied Mathematics- Statistics in 2003 from Iran University of Science and Technology. He has a B.Sc. In Mathematics from Tehran Shahid Beheshti University, and MSc. in Mathematical Statistics from Iran University of Science and Technology.

E-mail: Yari@iust.ac.ir

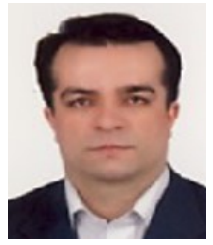

Alireza Sajedi is a Ph.D. student in the Department of Statistics at Iran Islamic Azad University of Science and Research branch (SRBIAU). His research focuses on entropy, fuzzy entropy, and portfolio selection using different entropy measures. Mr. Sajedi received his BSc. Degree in Statistics from Tehran Shahid Beheshti University in 2000, and his MSc. in Islamic Azad University North Tehran Branch in 2003.

E-mail: alireza.sajedi@srbiau.ac.ir

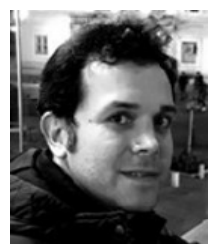

Mohamadtaghi Rahimi received his B.S. degree in statistics from Guilan university, Iran in 2008, and his Master and Ph.D degrees from the department of Mathematics and Statistics in Iran University of Science and Technology (IUST) in 2011 and 2016, respectively. He is currently a Post-Doctoral fellow in University of Northern Biritish Columbia (UNBC), BC, Canada. His research interests include fuzzy logic, fuzzy entropy and its application in labour market. E-mail: Mohamadtaghi.Rahimi@unbc.ca 